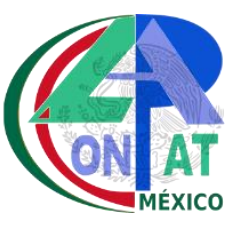

\title{
ELABORACIÓN DE MEZCLAS ASFÁLTICAS EN CALIENTE CON ADICIÓN DE RESIDUOS DE CONSTRUCCIÓN Y DEMOLICIÓN (CONCRETO HIDRÁULICO) EN UN 10\% Y $20 \%$ COMO SUSTITUCIÓN DE AGREGADO PÉTREO NATURAL
}

\author{
C. U. ESPINO GONZÁLEZ, C. LARA GÓMEZ, I. TRUJILLO CALDERÓN, \\ E. TAFOLLA MARTÍNEZ, E. A. NUÑEZ GUZMÁN. \\ Departamento de materiales, Facultad de Ingeniería Civil, \\ Universidad Michoacana de San Nicolás de Hidalgo, Morelia, Michoacán, México, 58070
}

\begin{abstract}
RESUMO
El objetivo fundamental de la investigación es la sustitución de agregados pétreos naturales por agregados de concreto reciclados lo que nos lleva a un ahorro ambiental y económico. La causa principal es que en México actualmente se generan 30 millones de toneladas de residuos de concreto hidráulico al año (Martínez Molina W., 2015). Para fines de esta investigación se implementó una sustitución de un $10 \%$ y un $20 \%$ de agregado pétreo natural por agregado de concreto reciclado. Finalmente los resultados comparativos entre la muestra testigo y los que contenían la sustitución del $10 \%$ y $20 \%$ son similares entre si arrojando resultados que nos brindan un cierto ahorro de material pétreo natural y nos brindan características similares a las de una mezcla asfáltica convencional.
\end{abstract}

Palavras clave: Reciclaje, Mezcla Asfáltica, Ahorro, Concreto.

\begin{abstract}
The fundamental objective of the research is the replacement of natural stone aggregates by aggregates of recycled concrete which leads to environmental and economic savings. The main cause is that in Mexico currently 30 million tons of hydraulic concrete waste are generated per year (Martínez Molina W., 2015). For the purposes of this research, a substitution of $10 \%$ and $20 \%$ of natural stone aggregate was implemented by adding recycled concrete. Finally, the comparative results between the control sample and those containing the $10 \%$ and $20 \%$ substitution are similar to each other, yielding results that give us a certain saving of natural stone material and give us characteristics similar to those of a conventional asphalt mixture.
\end{abstract}

Keywords: Recycling, Asphalt Mix, Saving, Concrete. 


\section{INTRODUCCIÓN.}

Hoy en día en la industria de la construcción hay demasiada generación de residuos a nivel mundial con una generación aproximada de 900 millones de toneladas al año (Martínez-Molina, 2015), en México generan tan solo en el Distrito Federal alrededor de 12,000 t/día de residuos sólidos urbanos, de los cuales el 50\% (6,000 t/día) son residuos de construcción y demolición (RCD) y solamente un porcentaje muy bajo es tratado (Martínez Daniel, 2013); siendo muy poca la gestión de estos residuos, esto ocasiona que la mayor parte de los mismos acabe en la vía pública, en tiraderos clandestinos, a orillas de las carreteras esto con los problemas que eso implica, y lo peor de todo es el desaprovechamiento de la vida útil que aún les queda. Los RCD están formados por diferentes materiales de construcción, entre los que por su composición y porcentaje de generación (65 al 75\% de los RCD) destacan los materiales de origen pétreo (concretos, morteros, ladrillos, azulejos, losetas, etc.), composición que los hace valorizables y por lo tanto adecuados para su retorno al ciclo de vida de la construcción.

El desarrollo sustentable en el sector de la construcción y en particular en la gestión de los RCD ha sido muy pobre en México, sin embargo es una necesidad que la sociedad demanda con una preocupación creciente sobre los sistemas tradicionales de eliminación de residuos y sus consecuencias para el medioambiente. Los objetivos principales medioambientales de la reducción, reutilización, reciclado y valorización de RCD se centran principalmente en la gestión de los RCD, donde el objetivo principal debe ser el minimizar su generación y en caso de existir, buscar su reciclaje o reaprovechamiento; siendo una etapa fundamental de este proceso de reciclaje la recogida selectiva, posteriormente adecuado proceso de transformación del residuo al agregado reciclado. Debido al gran impacto que la industria de la construcción genera a nivel mundial y al finalizar su vida útil no se leda un adecuado encausamiento a los RCD surge la necesidad de incorporar nuevas tendencias en la gestión de RCD, con un mayor respeto al medio ambiente, implementando técnicas de procesamiento, que ayuden a la reducción del volumen de desechos, en el número de rellenos sanitarios, explotación de recursos vírgenes, emisiones de $\mathrm{CO} 2$ al ambiente, generando así posibilidades de crecimiento para la industria del reciclaje.

Para mejorar la calidad del árido reciclado se recomienda usar como primera opción concreto hidráulico proveniente de la infraestructura vial como los pavimentos rígidos.

Algunas investigaciones marcan que las mezclas realizadas con árido natural presentan un comportamiento adecuado a la acción del agua, cumpliendo los requisitos establecidos para el ensayo de inmersión-compresión, mientras que las mezclas realizadas con un 50\% de árido reciclado presentan una pérdida de resistencia excesiva (Pérez, et al. 2007).

Por otra parte, los valores del módulo dinámico son razonables, siendo mayores en mezclas con árido reciclado al 50\% (Pérez, et al. 2007).

Además, se ha podido llevar a cabo una dosificación de mezclas bituminosas que contienen árido reciclado en un 50\% de peso en todas las fracciones. Estas mezclas tienen un contenido de betún superior al de las mezclas realizadas sólo con árido natural y además necesitan una mayor cantidad de filler, y sólo así se han podido cumplir los requisitos que el PG-3 establece para los parámetros obtenidos en el ensayo Marshall en carreteras de bajo tráfico. Por otro lado, no se ha podido realizar una dosificación de mezclas realizadas únicamente con árido reciclado (Pérez, et al. 2007).

\section{PROCEDIMIENTO.}

Para esta investigación se aplicó la metodología Marshall (Humboldt Mfg. Co., 2019) para la elaboración de mezclas asfálticas con sustitución en porcentaje de $10 \%$ y $20 \%$ de concreto reciclado. A continuación, se muestra la metodología de ensayo que se siguió para la obtención de los resultados. 


\subsection{Metodología de ensayos}

1. Caracterización de agregados gruesos.

2. Caracterización de agregados finos.

3. Caracterización del asfalto.

4. Diseño de las Mezclas Asfálticas con agregados RCD.

La realización del programa experimental consta de 2 etapas:

I. Primera etapa: Obtención y estudio de las propiedades físico-mecánicas de los agregados reciclados, que serán comparadas con las de los agregados naturales seleccionados para realizar las diversas experimentaciones.

2. Segunda etapa: Se planteará el uso de gruesos y finos en la composición de una mezcla asfálticas densa para carpeta de rodadura con la adición de un porcentaje controlado de árido reciclado. Así como el diseño volumétrico para la obtención del contenido óptimo de asfalto para cada uno de los porcentajes, la prueba testigo, con 10\% de agregado reciclado, $20 \%$ y $30 \%$ mediante la metodología Marshall.

Se recomienda para futuras investigaciones evaluar la susceptibilidad a la humedad de la mezcla asfáltica con agregado reciclado, ya que es importante determinar este parámetro que es la resistencia al paso de agua y aire hacia el interior, o a través del concreto asfáltico. La resistencia al daño por humedad se relaciona con las propiedades químicas del agregado mineral y el contenido de vacíos de aire en la mezcla compactada, y por tanto con los procesos de oxidación del asfalto, su adherencia y el drenaje del pavimento. (4).

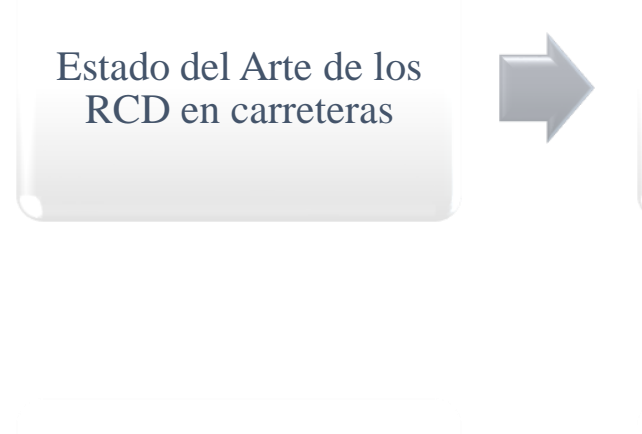

Analisis y discusión de Resultados.
Selección y analisis de los materiales mediante normativa mexicana

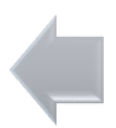

Diseño mediante Metodología Marshall para $20 \%$ con RCD
Diseño mediante Metodología Marshall con $100 \%$ agregado natural

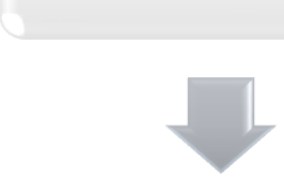

Diseño mediante Metodología Marshall para $10 \%$ con RCD

Figura 1

\section{RESULTADOS.}

A continuación, se muestran los resultados de los diseños que se compararan lo que es la mezcla testigo con agregado $100 \%$ natural, $10 \%$ agregado reciclado y $20 \%$ agregado reciclado como sustitución del agregado natural. Para el diseño todos los diseños se diseñará para un tráfico de más de un millón de ejes equivalentes y hasta diez millones de ejes equivalentes el cual tiene que cumplir con las especificaciones marcadas por la normativa de la SCT (CMT - Características de los Materiales, 2008) por el método de diseño Marshall. 
El material en estudio es proveniente de la región de Morelia, Michoacán con un tamaño máximo nominal de $3 / 4$ "; estos agregados pétreos naturales fueron obtenidos del banco de material llamado la Roka ubicado en la carretera Morelia - Uriangato 634, Cuto del Porvenir, Mich, México los cuales son producto de la trituración de las canteras cercanas del banco es material 100\% triturado tanto la grava, sello y arena.

A continuación, se presentan las diferentes gráficas comparativas de resultados.

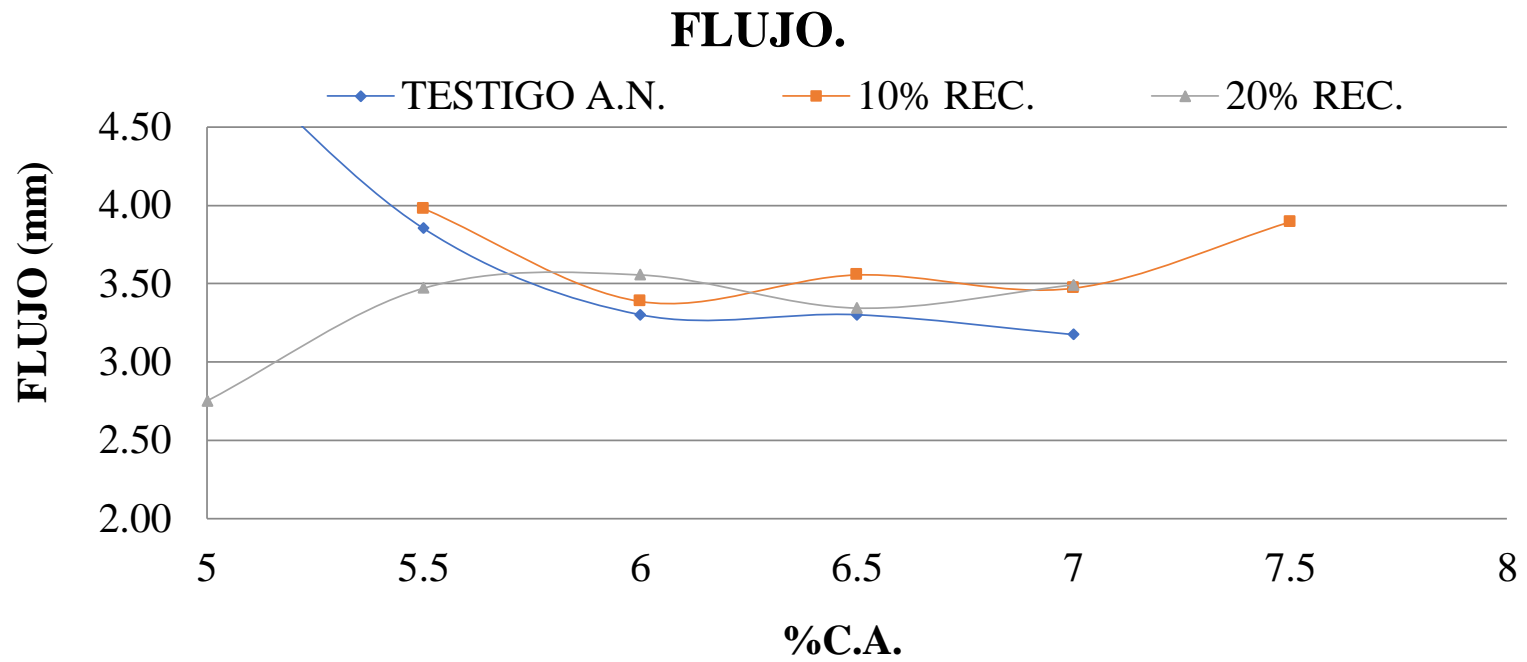

Figura 2

En la gráfica de flujo se puede observar que para los tres diseños el flujo se mantiene dentro de los límites establecidos por la normativa de la secretaria de comunicaciones y transportes, aunque ligeramente se observó un poco más de flujo para los diseños con agregado reciclado.

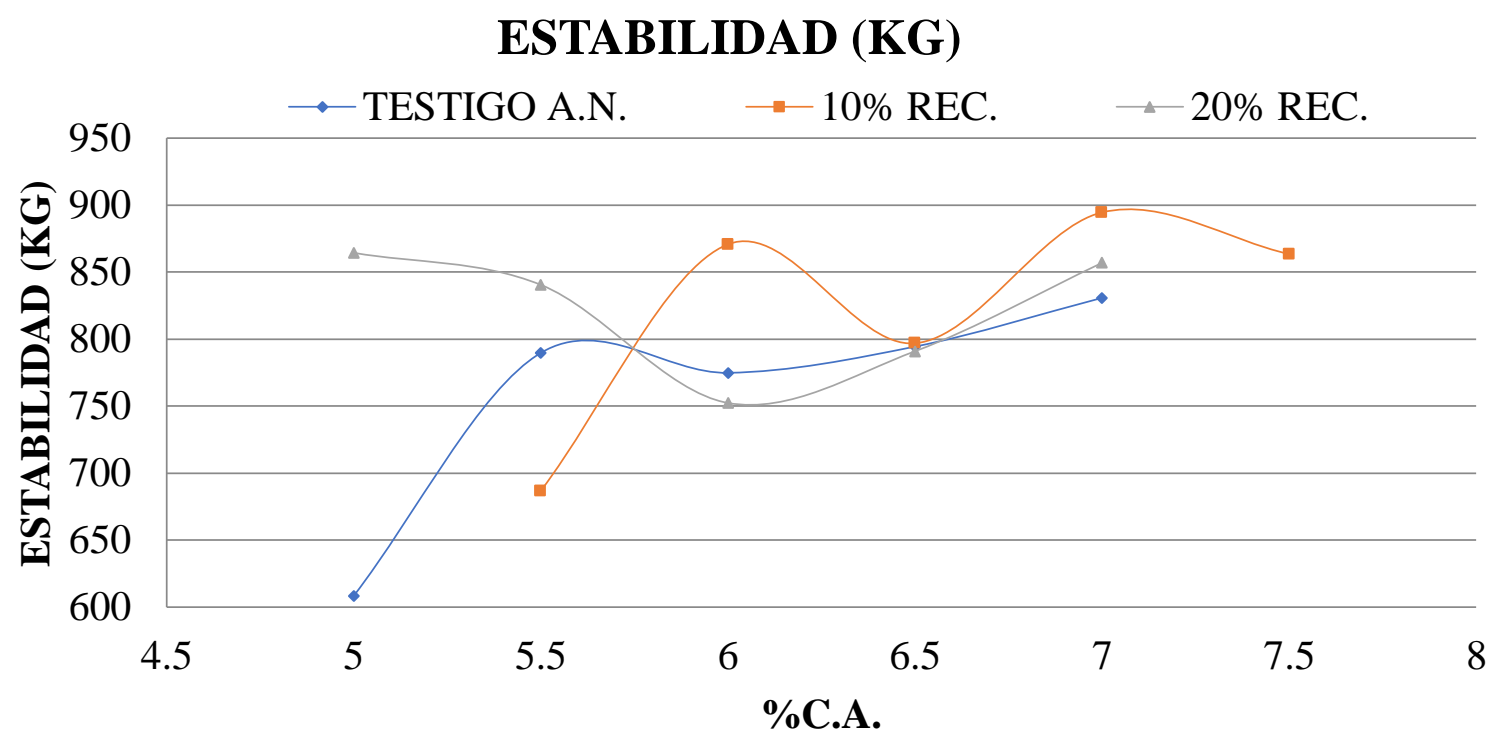

Figura 3 
En la gráfica de estabilidad se puede observar que el diseño para $10 \%$ y $20 \%$ tiene una cierta mejora que el diseño testigo con agregado $100 \%$ natural lo cual es benéfico para fines de ahorro económico y del medio ambiente, así como para su durabilidad.

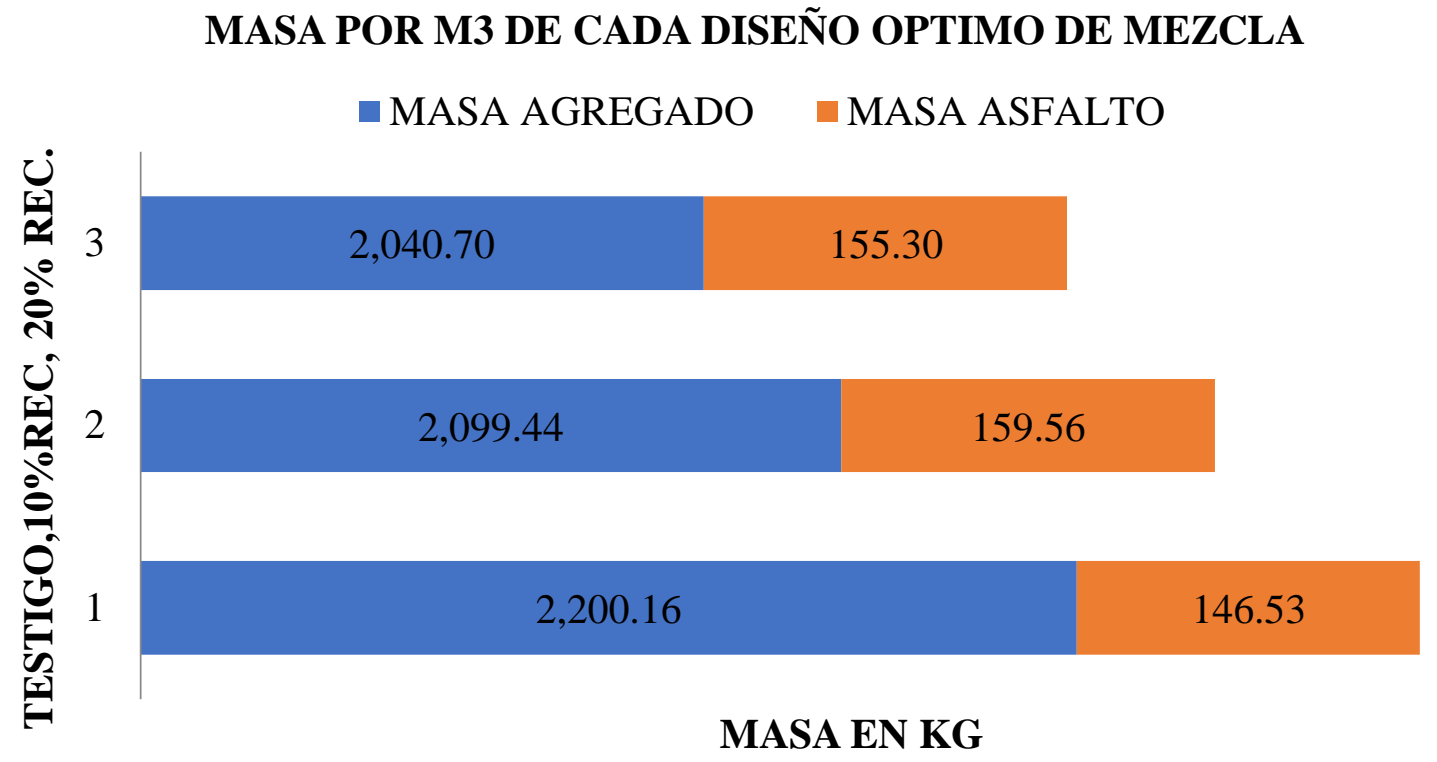

Figura 4

En la figura 4 se hace un análisis comparativo donde:

1 es el diseño óptimo con agregado pétreo $100 \%$ natural.

2 es el diseño óptimo con agregado pétreo $90 \%$ natural $10 \%$ reciclado.

3 es el diseño óptimo con agregado pétreo $80 \%$ natural $20 \%$ reciclado

1. La masa requerida de agregado por $\mathrm{m} 3$ va disminuyendo conforme va aumentando el agregado de RCD, por lo que esto podría significar un ahorro.

2. El asfalto primero aumenta en el diseño de $10 \%$ y $20 \%$ debido a que el asfalto absorbido aumenta entonces se requiere más asfalto para alcanzar el porcentaje de VAM, VAF y Vacíos en el agregado mineral. 


\section{M3 DE MEZCLA ASFALTICA POR DISEÑO}

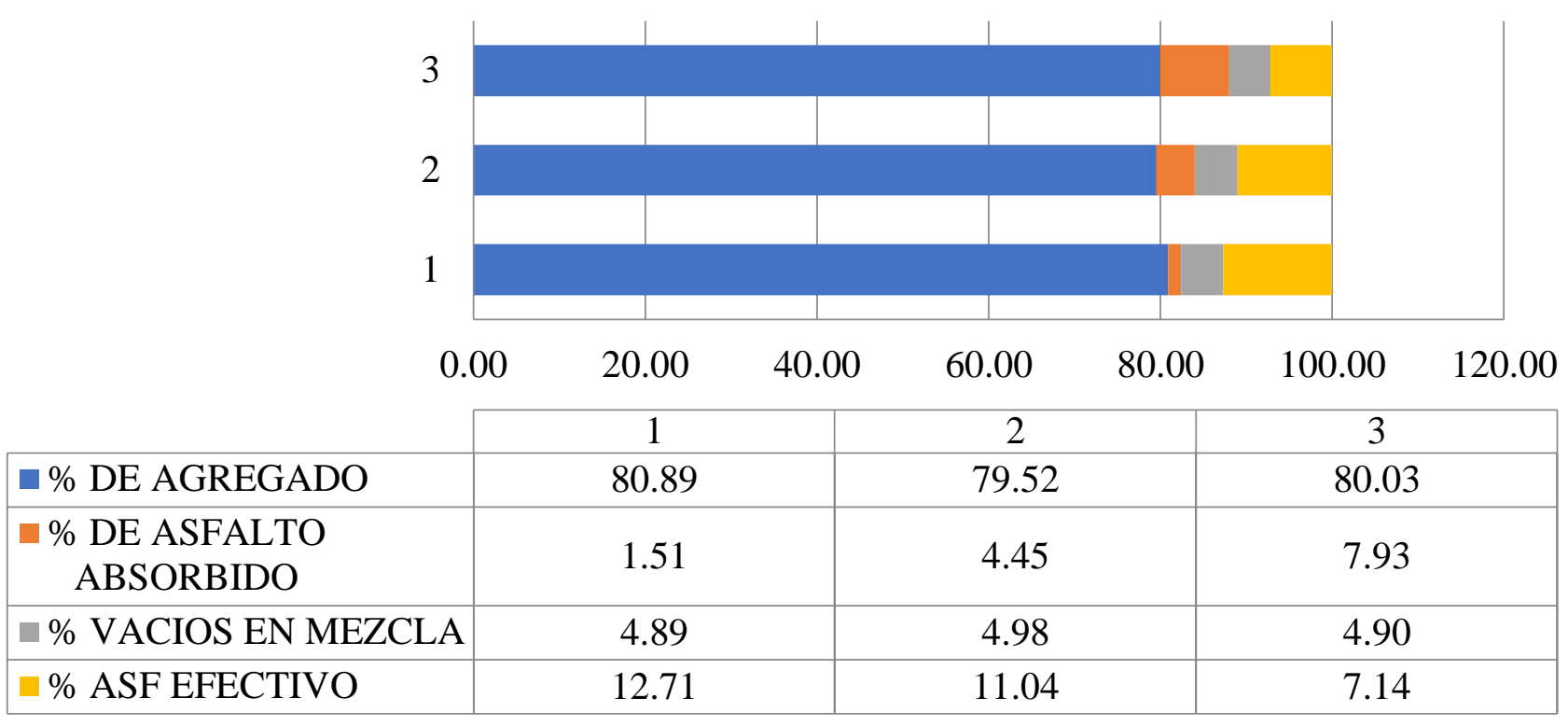

Figura 5

En la figura 5 se trata de hacer un contraste entre los diferentes diseños óptimos mostrando el porcentaje de agregado, de asfalto y de vacíos. Donde:

1 es el diseño de la mezcla con agregado pétreo $100 \%$ natural.

2 es el diseño de la mezcla con agregado pétreo $90 \%$ natural y $10 \%$ reciclado.

3 es el diseño de la mezcla con agregado pétreo $80 \%$ natural $20 \%$ reciclado.

Así podemos observar que en cuanto al volumen de agregado va paulatinamente aumentado conforme se añade mas RCD, el asfalto absorbido al igual se muestra que va aumentando y por lo tanto el efectivo disminuye y finalmente los vacíos en la mezcla se mantienen en el mismo porcentaje aproximadamente para los 3 diseños.

\section{CONCLUSIONES.}

Las mezclas asfálticas con $10 \%$ y $20 \%$ de agregado reciclado presentan un peso específico menor a medida que se va aumentando el agregado reciclado lo cual es debido a la densidad del mismo agregado reciclado que es más baja que el agregado natural.

Para el análisis de la gráfica de flujo podemos observar que es muy similar el comportamiento para los 3 diferentes muestras con distintos porcentajes de RCD la diferencia más significativa que se observa es que las muestras con $100 \%$ agregado natural fluye ligeramente más que con $10 \%$ y $20 \%$ de RCD y esto se podría deber a la forma de la partícula del agregado $100 \%$ natural que es un poco más plana y con menos caras fracturadas por el proceso de trituración; en cambio con $10 \%$ y $20 \%$ de RCD tiene menor deformación a menor contenido asfalto debido a que hay más porcentaje de caras fracturadas y el anclaje entre asfalto-agregado es mejor por los poros del RCD.

Finalmente, la estabilidad mejora conforme se va añadiendo agregado de concreto hidráulico debido a que el concreto reciclado presenta mayor rigidez que el agregado natural, lo cual se traduce en una mejor estabilidad de la mezcla asfáltica con $10 \%$ y $20 \%$ de agregado reciclado. 


\section{REFERENCIAS.}

CMT - Características de los Materiales (2008), Calidad de mezclas asfálticas para carreteras. Capítulo 3, Título 5: Materiales asfálticos, aditivos y mezclas. Parte 4: Materiales para pavimentos. NCTM-4-05-003/08

Garnica Anguas, P., M. Flores Flores, J. Gómez López, Delgado Alamilla, H. (2005). Caracterización geomecánica de mezclas asfálticas, publicación no. 267, Instituto Mexicano del Transporte, Sanfandila, Querétaro.

Humboldt Mfg. Co. (2019), Marshall Mix Design and Testing Booklet. Consultar en: www.humboldtmfg.com

Martínez-Molina, W., Torres-Acosta, A., Alonso-Guzmán, E., Chávez-García, H., Hernández-Barrios, H., Lara-Gómez, C., Martínez-Alonso, W., Pérez-Quiroz, J., Bedolla-Arroyo, J., González-Valdéz, F. (2015). Concreto reciclado: una revisión. Revista ALCONPAT, 5(3), 224 - 237. doi: http://dx.doi.org/10.21041/ra.v5i3.91

Martínez Daniel, I. (2013), Residuos de construcción y demolición (RCD): Situación actual y correcta gestión para el proceso de reciclaje en la industria mexicana. Tesis de Maestría. Universidad Nacional Autónoma de México. http://132.248.9.195/ptd2013/julio/0696879/Index.html

Pérez, I., Toledano, M., Gallego, J., Taibo, J. (2007). Propiedades mecánicas de mezclas bituminosas en caliente fabricadas con áridos reciclados de residuos de construcción y demolición. Materiales de Construcción, 57(285), 17-29. doi: http://dx.doi.org/10.3989/mc.2007.v57.i285.36 